\title{
FINITE STRAIN FORMULATION OF ELASTO-PLASTICITY WITHOUT YIELD SURFACE: THEORY, PARAMETER IDENTIFICATION AND APPLICATIONS
}

\author{
Cyprian Suchocki, Pawee Skoczylas \\ Warsaw University of Technology, Department of Mechanics and Armament Technology, Warsaw, Poland \\ e-mail: c.suchocki@imik.wip.pw.edu.pl
}

\begin{abstract}
In this study, a finite strain formulation of elasto-plasticity without the yield surface is analyzed. It is found that the presented model allows for a good description of the elasto-plastic response for many metallic materials. Furthermore, it is noted that the model involving a limited number of material parameters is able to capture such effects as indefinite elastic limit, strain hardening, strain softening and the Bauschinger effect. An algorithm for the evaluation of constants is described and the determined values are given for NC high carbon tool steel, copper, brass, Inconel alloy, tungsten heavy alloy and aluminum.
\end{abstract}

Keywords: constitutive equation, plasticity, metals, material constants

\section{Introduction}

In continuum mechanics, two main concepts of the plasticity theory can be distinguished, i.e. flow theories and theories of plasticity without the yield surface, including endochronic plasticity. The origins of constitutive theories based on the flow rules date back to the mid-19th century. The fundamentals of plasticity theories not employing the yield surface were formulated much later (e.g. Ilyushin, 1963; Pipkin and Rivlin, 1965).

The first steps associated with replacing the flow rule and yield criterion-based formulation of elasto-plasticity with a new one are claimed to Ilyushin (1963) who first introduced the concept of arc length in the strain space. This approach was later extended by Pipkin and Rivlin (1965) who developed a novel, general theory of plasticity for the case of finite strains and rotations, however, without proposing a specific form of the constitutive equation.

The arc length calculated in nine-dimensional finite strain space is the basic concept for the new formulation of elasto-plasticity. This scalar value is utilized to build a functional of strain history analogous to the history functional used in the theory of viscoelasticity. Pipkin and Rivlin (1965) considered the most general form of the strain history functional, which in practice can be assumed in the form of a hereditary integral, where, instead of time, the arc length in the strain space is assumed as the variable of integration. For a constitutive theory constructed in such a manner, the arc length plays the role of an intrinsic time analogously to the time in the theory of viscoelasticity.

Pipkin and Rivlin (1965) postulated that the arc length should be calculated utilizing a Pythagorean metric. A different approach was introduced by Valanis (1971a,b) who proposed using a non-Pythagorean metric for calculation of the arc length in the infinitesimal strain space and additional multiplication by a scaling function. The plasticity theories which employ a scaling function in their definition of the intrinsic time are called endochronic (cf. Valanis, 1971b). Such concepts were strongly criticized (e.g. Rivlin, 1981) as nonphysical. Some of the imperfections of the original endochronic theory were eliminated in a later constitutive model 
(cf. Valanis, 1980). However, this was achieved at the price of splitting the strain tensor into elastic and plastic components and introducing a yield criterion.

A number of models exist which transfer some concepts of the endochronic formulation to the flow theory. In particular, the flow rule for kinematic hardening may utilize a hereditary integral to govern the evolution of the back stress (e.g. Backhaus, 1976). This framework was further extended to take into account such effects as the memory of maximal prestrain and the amplitude-dependent hardening in the case of cyclic loadings (cf. Mróz and Rodzik, 1996). The effective infinitesimal plastic strain is used as the intrinsic time in models of this type.

In the present work, a finite strain formulation of plasticity without the yield surface, previously introduced by Suchocki (2015), is analyzed upon its ability to capture the inelastic response of metals. In order to clearly lay out basic theory assumptions, the infinitesimal strain formulation is described first. Subsequently, the novel finite strain generalization is presented and examined. It is found that the proposed constitutive model, which involves a very limited number of material parameters, is not only able to accurately fit the stress-strain data but also to predict such behavior as indefinite elastic limit, strain hardening, strain softening and the Bauschinger effect. A numerical algorithm used for the evaluation of the material constants is described. The model parameters are determined for a variety of metallic materials. Finally, a parameric study is conducted.

\section{Small strain formulation}

Below, the basic notions of rate-independent plasticity are discussed in the case of infinitesimal deformations, cf. Rivlin (1981). The following assumptions have been adopted:

- Only isothermal deformation processes are considered.

- Isotropic materials with elasto-plastic properties are analyzed.

- The volumetric response is purely elastic (Fig. 1a).

- The total shear stress is assumed as a sum of the elastic stress and $N$ rate-independent stress-like internal state variables (Fig. 1b).

- The internal state variables depend on shear deformation only and are governed by differential evolution equations.

(a)

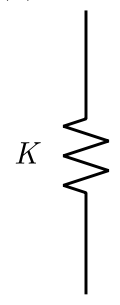

(b)

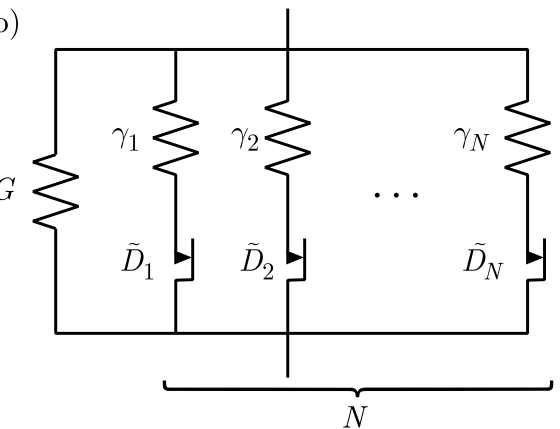

Fig. 1. Mechanical scheme of the constitutive model; (a) volumetric response, (b) shear response

The total Cauchy stress tensor $\boldsymbol{\sigma}$ is a sum of the volumetric stress tensor $p \mathbf{1}$ and the stress deviator s, i.e.

$$
\boldsymbol{\sigma}=p \mathbf{1}+\mathbf{s} \quad p=K \epsilon \quad \mathbf{s}=\mathbf{s}_{0}+\sum_{k=1}^{N} \widetilde{\mathbf{h}}_{k} \quad \mathbf{s}_{0}=2 G \mathbf{e}
$$


where

$$
\epsilon=\operatorname{tr} \varepsilon \quad \mathbf{e}=\varepsilon-\frac{1}{3}(\operatorname{tr} \varepsilon) \mathbf{1}
$$

whereas $\widetilde{\mathbf{h}}_{k}(k=1,2, \ldots, N)$ are tensorial internal state variables while $K$ and $G$ are the bulk and shear moduli, respectively. The internal state variable evolution equation is a modification of the differential equation following from the Maxwell mechanistic model, i.e.

$$
\dot{\widetilde{\mathbf{h}}}_{k}+\frac{1}{\widetilde{D}_{k} M(|\dot{\mathbf{e}}|)} \widetilde{\mathbf{h}}_{k}=\gamma_{k} \dot{\mathbf{s}}_{0} \quad M(|\dot{\mathbf{e}}|)=\left(\operatorname{tr} \dot{\mathbf{e}}^{2}\right)^{-\frac{1}{2}} \quad k=1,2, \ldots, N
$$

where $\gamma_{k}$ is a dimensionless parameter and the product $\widetilde{D}_{k} M(|\dot{\mathbf{e}}|)$ is a rate-dependent relaxation time. The particular choice of $M(|\dot{\mathbf{e}}|)$ given by $(2.3)_{2}$ allows one to eliminate time from the evolution equation. After inserting Eqs $(2.1)_{4}$ and $(2.3)_{2}$ into Eq. $(2.3)_{1}$ and multiplying it by a time differential, an incremental equation is obtained

$$
\mathrm{d} \widetilde{\mathbf{h}}_{k}+\frac{\sqrt{\operatorname{tr} \mathrm{d} \mathbf{e}^{2}}}{\widetilde{D}_{k}} \widetilde{\mathbf{h}}_{k}=2 \gamma_{k} G \mathrm{~d} \mathbf{e} \quad k=1,2, \ldots, N
$$

which states that the evolution of $\widetilde{\mathbf{h}}_{k}(k=1,2, \ldots, N)$ depends solely on the deformation path in the strain space and is rate-independent.

An intrinsic time $\zeta$ is introduced, cf. Valanis $(1971 a, b)$, i.e.

$$
\dot{\zeta}=\frac{1}{M(|\dot{\mathbf{e}}|)} \quad \zeta(\tau)=\int_{-\infty}^{\tau} \frac{\mathrm{d} \tau^{\prime}}{M\left(\tau^{\prime}\right)} \quad \zeta(t)=z
$$

After substituting Eq. $(2.3)_{2}$ into Eq. $(2.5)_{2}$

$$
\zeta(\tau)=\int_{-\infty}^{\tau} \sqrt{\mathrm{de}\left(\tau^{\prime}\right) \cdot \mathrm{d} \mathbf{e}\left(\tau^{\prime}\right)}
$$

It can be seen from (2.6) that the intrinsic time has an interpretation of the arc length in the deviatoric strain space. Integration of Eq. $(2.3)_{1}$ yields

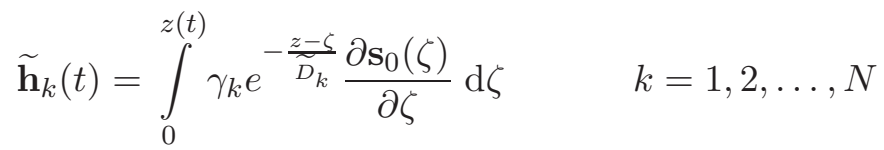

which is reminiscent of a similar equation present in both linear and nonlinear viscoelasticity.

\section{Finite strain formulation}

All the basic assumptions listed in the previous Section apply to the finite strain model aswell. The total stress $\mathbf{S}$ is given as a sum of the volumetric stress $\mathbf{S}_{0}^{v o l}$, elastic isochoric stress $\mathbf{S}_{0}^{i s o}$ and the stress-like tensorial state variables $\widetilde{\mathbf{H}}_{k}(k=1,2, \ldots, N)$, i.e.

$$
\mathbf{S}=\mathbf{S}_{0}^{v o l}+\mathbf{S}_{0}^{i s o}+\sum_{k=1}^{N} \widetilde{\mathbf{H}}_{k} \quad \mathbf{S}_{0}^{v o l}=J \frac{\partial U}{\partial J} \mathbf{C}^{-1} \quad \mathbf{S}_{0}^{i s o}=2 J^{-\frac{2}{3}}\left[\frac{\partial \bar{W}}{\partial \overline{\mathbf{C}}}\right]
$$

where, $\mathbf{C}$ and $\overline{\mathbf{C}}$ are the total and isochoric right Cauchy-Green $(\mathrm{C}-\mathrm{G})$ tensors, respectively, $J=\operatorname{det} \mathbf{F}$ is the determinant of the deformation gradient tensor $\mathbf{F}$, whereas $U=U(J)$ and 
$\bar{W}=\bar{W}\left(\bar{I}_{1}, \bar{I}_{2}\right)$ are the elastic stored energy functions with $\bar{I}_{1}$ and $\bar{I}_{2}$ being scalar invariants of $\overline{\mathbf{C}}$ while $\operatorname{DEV}[\bullet]=[\bullet]-\frac{1}{3}([\bullet] \cdot \overline{\mathbf{C}}) \overline{\mathbf{C}}^{-1}$. The evolution of internal state variables is governed by differential equations

$$
\dot{\widetilde{\mathbf{H}}}_{k}+\frac{1}{\widetilde{D}_{k} M(|\dot{\mathbf{C}}|)} \widetilde{\mathbf{H}}_{k}=\gamma_{k} \dot{\mathbf{S}}_{0}^{i s o} \quad k=1,2, \ldots, N
$$

with

$$
M(|\dot{\mathbf{C}}|)=\bar{J}_{2}^{-\frac{1}{2}} \quad \bar{J}_{2}=\operatorname{tr} \dot{\overline{\mathbf{C}}}^{2}
$$

This specific choice of $M(|\dot{\overline{\mathbf{C}}}|)$ ensures that the evolution of $\widetilde{\mathbf{H}}_{k}(k=1,2, \ldots, N)$ is rateindependent. Integration of (3.2) yields

$$
\widetilde{\mathbf{H}}_{k}(t)=\int_{0}^{z(t)} \gamma_{k} \mathrm{e}^{-\frac{z-\zeta}{\widetilde{D}_{k}}} \frac{\partial \mathbf{S}_{0}^{i s o}(\zeta)}{\partial \zeta} \mathrm{d} \zeta \quad k=1,2, \ldots, N
$$

where the following equalities are used

$$
\dot{\zeta}=\frac{1}{M(|\dot{\mathbf{C}}|)} \quad \zeta(\tau)=\int_{-\infty}^{\tau} \sqrt{\mathrm{d} \overline{\mathbf{C}}\left(\tau^{\prime}\right) \cdot \mathrm{d} \overline{\mathbf{C}}\left(\tau^{\prime}\right)} \quad \zeta(t)=z
$$

Equation (3.4) is a special case of the general, functional stress-strain relation formulated by Pipkin and Rivlin (1965), and the intrinsic time introduced in Eqs (3.5) is a modification of the fictitious time measure proposed therin.

\section{One-dimensional tension-compression}

A uniaxial tension along the $X_{1}$-direction (Fig. $2 \mathrm{~b}$ ) is defined by the following set of equations

$$
x_{1}=\lambda_{1} X_{1} \quad x_{2}=\lambda X_{2} \quad x_{3}=\lambda X_{3}
$$

where the principal stretches $\lambda_{1}>1$ and $\lambda<1$ for tension, whereas $\lambda_{1}<1$ and $\lambda>1$ for compression processes. Applying the boundary condition of the stress-free lateral surface, the scalar equations can be written as follows

$$
S_{11}=S_{0(11)}^{v o l}+S_{0(11)}^{i s o}+\sum_{k=1}^{N} \widetilde{H}_{k(11)} \quad 0=S_{0(22)}^{v o l}+S_{0(22)}^{i s o}+\sum_{k=1}^{N} \widetilde{H}_{k(22)}
$$

Is is assumed that $\bar{W}(\overline{\mathbf{C}})=\bar{W}\left(\bar{I}_{1}\right)$, which yields

$$
\mathbf{S}_{0}^{v o l}=J p \mathbf{C}^{-1} \quad p=\frac{\partial U}{\partial J} \quad \mathbf{S}_{0}^{i s o}=2 J^{-\frac{2}{3}} \frac{\partial \bar{W}}{\partial \bar{I}_{1}}\left(\mathbf{1}-\frac{1}{3} I_{1} \mathbf{C}^{-1}\right)
$$

In the considered case $I_{1}=\lambda_{1}^{2}+2 \lambda^{2}$, which leads to the following formulas for the isochoric elastic stress components

$$
S_{0(11)}^{i s o}=\frac{4}{3} J^{-\frac{2}{3}} \frac{\partial \bar{W}}{\partial \bar{I}_{1}}\left[1-\left(\frac{\lambda}{\lambda_{1}}\right)^{2}\right] \quad S_{0(22)}^{i s o}=-\frac{1}{2}\left(\frac{\lambda_{1}}{\lambda}\right)^{2} S_{0(11)}^{i s o}
$$


By utilizing Eq. (4.3) 1 in Eqs (4.2), one obtains

$$
S_{11}=J p \frac{1}{\lambda_{1}^{2}}+S_{0(11)}^{i s o}+\sum_{k=1}^{N} \widetilde{H}_{k(11)} \quad 0=J p \frac{1}{\lambda^{2}}+S_{0(22)}^{i s o}+\sum_{k=1}^{N} \widetilde{H}_{k(22)}
$$

After eliminating the $J p$ factor, and using $(4.4)_{2}$, the following form of the process equation is found

$$
S_{11}=\frac{3}{2} S_{0(11)}^{i s o}+\sum_{k=1}^{N}\left[\widetilde{H}_{k(11)}-\left(\frac{\lambda}{\lambda_{1}}\right)^{2} \widetilde{H}_{k(22)}\right]
$$

In this study, two specific forms of $\bar{W}$ are considered, i.e. neo-Hooke and the one proposed by Knowles

$$
\bar{W}\left(\bar{I}_{1}\right)=\frac{\mu}{2}\left(\bar{I}_{1}-3\right) \quad \bar{W}\left(\bar{I}_{1}\right)=\frac{\mu}{2 b}\left\{\left[1+\frac{b}{\kappa}\left(\bar{I}_{1}-3\right)\right]^{\kappa}-1\right\}
$$

with $\mu, b$ and $\kappa$ being the elasticity constants. After inserting $(4.7)_{1}$ into $(4.4)_{1}$, the following formula is found

$$
S_{0(11)}^{\text {iso }}=\frac{2}{3} J^{-\frac{2}{3}} \mu\left[1-\left(\frac{\lambda}{\lambda_{1}}\right)^{2}\right]
$$

whereas substituting $(4.7)_{2}$ into $(4.4)_{1}$ yields

$$
S_{0(11)}^{i s o}=\frac{2}{3} J^{-\frac{2}{3}} \mu\left[1+\frac{b}{\kappa}\left(\bar{I}_{1}-3\right)\right]^{\kappa-1}\left[1-\left(\frac{\lambda}{\lambda_{1}}\right)^{2}\right]
$$

In the case of material incompressibility $J=1$ and $\lambda=\lambda_{1}^{-\frac{1}{2}}$, whereas $\bar{I}_{1}=I_{1}=\lambda_{1}+2 \lambda_{1}^{-\frac{1}{2}}$. Thus, Eqs (4.8) and (4.9) take respectively forms

$$
S_{0(11)}^{i s o}=\frac{2}{3} \mu\left(1-\frac{1}{\lambda_{1}^{3}}\right) \quad S_{0(11)}^{i s o}=\frac{2}{3} \mu\left[1+\frac{b}{\kappa}\left(I_{1}-3\right)\right]^{\kappa-1}\left(1-\frac{1}{\lambda_{1}^{3}}\right)
$$

By applying the incompressibility condition to Eq. (4.6), the one-dimensional process equation is found, i.e.

$$
S_{11}=\frac{3}{2} S_{0(11)}^{i s o}+\sum_{k=1}^{N}\left(\widetilde{H}_{k(11)}-\widetilde{H}_{k(22)} \frac{1}{\lambda_{1}^{3}}\right)
$$

whereas the engineering and Cuachy stresses are calculated according to the formulas

$$
T_{11}=\lambda_{1} S_{11} \quad \sigma_{11}=\lambda_{1} T_{11}
$$

Equations (4.10)-(4.12) along with Eq. (4.4) 2 and two scalar equations following from Eq. (3.2) form up a system of nonlinear algebraic and differential equations which can be solved numerically. It is assumed that the excitation is kinematic with a constant strain rate $T$, thus, at the time increment $n+1$

$$
t_{n+1}=t_{n}+\Delta t \quad \lambda_{1}\left(t_{n+1}\right)=1+T t_{n+1}
$$

In the case of incompressibility $\bar{J}_{2}=J_{2}=\operatorname{tr} \dot{\mathbf{C}}$. Furthermore, an auxiliary quantity is defined as

$$
\widetilde{J}\left(t_{n+1}\right)=J_{2}\left(t_{n+1}\right) \Delta t^{2}
$$




\section{Material parameter optimization algorithm}

Input: $\mathbf{p}^{0},\left(\widetilde{T}_{11}\right)_{j}, w_{j}(j=1,2, \ldots, K), N, \widetilde{T}, i_{\max }, i=1$

1. Calculate the inital value of the weighted error function

$$
\mathcal{F}^{0}\left(\mathbf{p}^{0}\right)=\sum_{j=1}^{K} w_{j}\left[\left(T_{11}\left(\mathbf{p}^{0}\right)\right)_{j}-\left(\widetilde{T}_{11}\right)_{j}\right]^{2}
$$

2. Assemble the initial matrix of parameter values

$$
\mathbf{M}_{5 \times(1+2 N)}^{0}=\left[\begin{array}{ccccccc}
\mu_{1} & \gamma_{11} & \cdots & \gamma_{N 1} & \widetilde{D}_{11} & \cdots & \widetilde{D}_{N 1} \\
\mu_{2} & \gamma_{12} & \cdots & \gamma_{N 2} & \widetilde{D}_{12} & \cdots & \widetilde{D}_{N 2} \\
\vdots & \vdots & \vdots & \vdots & \vdots & \vdots & \vdots \\
\mu_{5} & \gamma_{15} & \cdots & \gamma_{N 5} & \widetilde{D}_{15} & \cdots & \widetilde{D}_{N 5}
\end{array}\right]
$$

3. Find a parameter combination minimizing the error function

$$
\mathbf{p}^{i}=\left[\mu^{o p t}, \gamma_{1}^{o p t}, \ldots, \gamma_{N}^{o p t}, \widetilde{D}_{1}^{o p t}, \ldots, \widetilde{D}_{N}^{o p t}\right]^{\mathrm{T}}
$$

4. Check the stop criterion, i.e.

$$
\left|\frac{\mathcal{F}^{i}\left(\mathbf{p}^{i}\right)-\mathcal{F}^{i-1}\left(\mathbf{p}^{i-1}\right)}{\mathcal{F}^{i-1}\left(\mathbf{p}^{i-1}\right)}\right|<\widetilde{T}
$$

5. Assemble the matrix of parameter values for the next iteration

(a) Calculate parameter value increments $(k=1,2, \ldots, N)$

$$
\begin{aligned}
& \Delta \mathbf{p}=\left[\Delta \mu, \Delta \gamma_{1}, \ldots, \Delta \gamma_{N}, \Delta \widetilde{D}_{1}, \ldots, \Delta \widetilde{D}_{N}\right]^{\mathrm{T}} \\
& \Delta \mu=\frac{\left|\mu^{o p t}\right|}{5 i} \quad \Delta \gamma_{k}=\frac{\left|\gamma_{k}^{\text {opt }}\right|}{5 i} \quad \Delta \widetilde{D}_{k}=\frac{\left|\widetilde{D}_{k}^{\text {opt }}\right|}{5 i}
\end{aligned}
$$

(b) Compute $\mathbf{M}_{5 \times(1+2 N)}^{i}$ components $(k=1,2, \ldots, 5, l=1,2, \ldots, 1+2 N)$

$$
\left[\mathbf{M}_{5 \times(1+2 N)}^{i}\right]_{k l}=M_{k l}^{i}=p_{l}^{i}+\frac{k-3}{2} \Delta p_{l}
$$

6. Go to step 3 if $i<i_{\max }$; otherwise display optimal material parameter values

which is determined by the following formula

$$
\begin{aligned}
& \widetilde{J}\left(t_{n+1}\right)=2(T \Delta t)^{2}\left\{\frac{2}{3}\left[\frac{1}{3}\left(\lambda_{1}^{4}\left(t_{n+1}\right)+\frac{2}{\lambda_{1}^{2}\left(t_{n+1}\right)}\right)-2\right]\left(\lambda_{1}^{3}\left(t_{n+1}\right)-\frac{1}{\lambda_{1}^{3}\left(t_{n+1}\right)}\right)^{2}\right. \\
& \left.+2 \lambda_{1}^{2}\left(t_{n+1}\right)+\frac{1}{\lambda_{1}^{4}\left(t_{n+1}\right)}\right\}
\end{aligned}
$$

According to Eqs $(4.10)_{1}$ and $(4.4)_{2}$, the isochoric elastic stresses are given as

$$
S_{0(11)}^{i s o}\left(t_{n+1}\right)=\frac{2}{3} \mu\left(1-\frac{1}{\lambda_{1}^{3}\left(t_{n+1}\right)}\right) \quad S_{0(22)}^{i s o}\left(t_{n+1}\right)=-\frac{1}{2} \lambda_{1}^{3}\left(t_{n+1}\right) S_{0(11)}^{i s o}\left(t_{n+1}\right)
$$


The total second P-K stress is calculated using Eq. (4.11), assuming $N=1$, i.e.

$$
S_{11}\left(t_{n+1}\right)=\frac{3}{2} S_{0(11)}^{i s o}\left(t_{n+1}\right)+\widetilde{H}_{1(11)}\left(t_{n+1}\right)-\frac{1}{\lambda_{1}^{3}\left(t_{n+1}\right)} \widetilde{H}_{1(22)}\left(t_{n+1}\right)
$$

whereas the internal state variable components are calculated from the following recurrenceupdate formulas

$$
\begin{aligned}
\widetilde{H}_{1(11)}\left(t_{n+1}\right) & =\frac{\left(1-\frac{1}{2 \widetilde{D}_{1} \widetilde{J}^{-\frac{1}{2}}\left(t_{n+1}\right)}\right) \widetilde{H}_{1(11)}\left(t_{n}\right)+\gamma_{1}\left(S_{0(11)}^{i s o}\left(t_{n+1}\right)-S_{0(11)}^{i s o}\left(t_{n}\right)\right)}{1+\frac{1}{2 \widetilde{D}_{1} \widetilde{J}^{-\frac{1}{2}}\left(t_{n+1}\right)}} \\
\widetilde{H}_{1(22)}\left(t_{n+1}\right) & =\frac{\left(1-\frac{1}{2 \widetilde{D}_{1} \widetilde{J}^{-\frac{1}{2}}\left(t_{n+1}\right)}\right) \widetilde{H}_{1(22)}\left(t_{n}\right)+\gamma_{1}\left(S_{0(22)}^{i s o}\left(t_{n+1}\right)-S_{0(22)}^{i s o}\left(t_{n}\right)\right)}{\left(1+\frac{1}{2 \widetilde{D}_{1} \widetilde{J}^{-\frac{1}{2}}\left(t_{n+1}\right)}\right)}
\end{aligned}
$$

which have been developed utilizing the central difference method.

The given above set of discretized process equations in the form derived for an incompressible material is utilized within the parameter evaluation algorithm for computation of theoretical stress values. The assumption of material incompressibility has been adopted for the sake of simplicity. The relative error in estimation of the stretch ratio in the lateral direction that results from this assumption does not exceed $2 \%$ for the considered class of materials.

\section{Experimetal setup}

A number of experiments have been performed in order to obtain the data which allowed one to asses the model's capability of capturing the mechanical response of metals. The Instron 1115 material testing machine was used for the experiments (Fig. 2a).

(a)

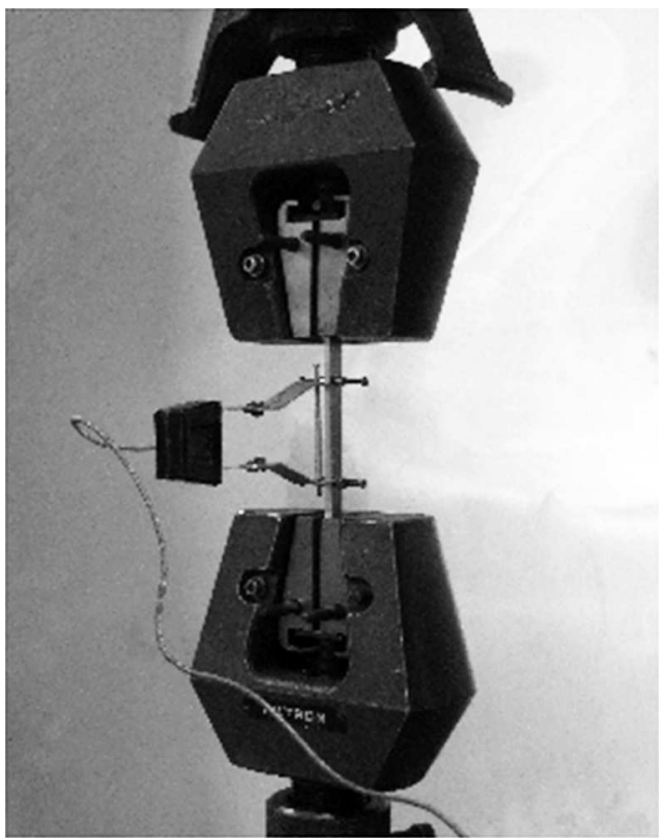

(b) $\quad x_{1}, X_{1} \Lambda$

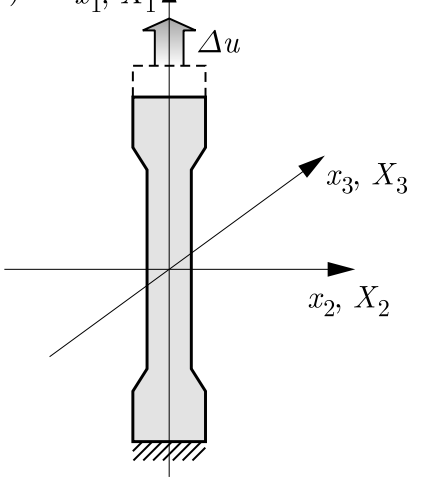

(c)

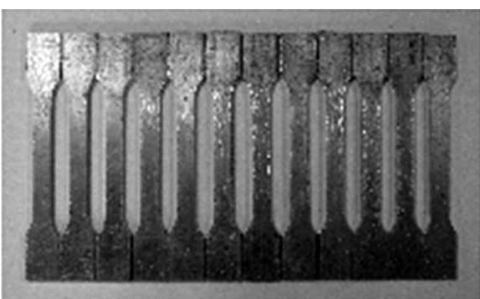

Fig. 2. (a) Experimental set-up. (b) Deformation process and coordinate systems. (c) Dogbone specimens

The conducted tests included one-dimensional tension (Fig. 2b) of copper, Inconel No. 1 (supersaturated), Inconel No. 2 (supersaturated and aged), brass, NC tool steel, aluminum and 
tungsten heavy alloy (THA). In each case, unloading was performed shortly before the failure. The specimens (Fig. 2c) were machined according to the Polish standard PN-91 H-04310. Each tension test was repeated for at least three times in order to make sure that the registered stress-strain curves were reproducible. The specimens were tested at room temperature of $20^{\circ} \mathrm{C}$.

\section{Curve fitting}

A pattern search algorithm has been used for the evaluation of the model parameters. It is a modification of the scheme proposed by Zalewski (2009). In each iteration, a matrix $\mathbf{M}_{5 \times(1+2 N)}^{i}$ containing several sets of material constants is assembled. Every parameter set is subsequently used to compute theoretical stress values. A combination of constant values $\mathbf{p}^{i+1}$, which results in the best approximation of the stress-stretch curve, is allowed to proceed to the next iteration $i+1$. The performed calculations have been listed in an outline, which assumed making use of neo-Hooke stored-energy function. The curve fitting results are presented in Figs. 3 and 4, whereas the evaluated values of the material parameters are gathered in Table 1.

Table 1. Determined values of material parameters

\begin{tabular}{|l|c|c|c|c|c|}
\hline \multicolumn{1}{|c|}{ Material } & $\mu[\mathrm{MPa}]$ & $b[-]$ & $\kappa[-]$ & $\gamma_{1}[-]$ & $\widetilde{D}_{1}[-]$ \\
\hline \hline copper & 367.33 & 22.41 & 0.68 & 132.80 & 0.0053 \\
\hline Inconel No. 1 & 1114.68 & - & - & 83.43 & 0.0034 \\
\hline Inconel No. 2 & 1152.58 & - & - & 74.12 & 0.013 \\
\hline brass & 681.68 & - & - & 35.67 & 0.013 \\
\hline steel NC 6 & 1065.19 & - & - & 47.06 & 0.0081 \\
\hline aluminum & 546.70 & - & - & 30.72 & 0.0098 \\
\hline THA & 1226.94 & - & - & 88.48 & 0.0060 \\
\hline
\end{tabular}

In each case, a single internal state variable has been assumed. In the case of copper, the version of the constitutive equation employing the Knowles stored-energy function has been used in order to reproduce the strain-softening phenomenon (Fig. 3a). For all the remaining metals, the neo-Hooke function has been utilized.

An excellent agreement between the theoretical and experimental curves has been achieved for THA (Fig. 4a). In some cases, fitting monotonic stress-stretch data leads to obtaining a better approximation as it can be seen in the case of brass (Fig. 4b).

\section{Parametric studies}

Several simulations have been carried out in order to asses the model behavior, its sensitivity and the influence of the experimental data used for material parameter evaluation.

In Fig. 5a, the results of loading-unloading simulations are presented which have been performed for five different values of the maximum stretch. The material parameter set has been evaluated from the brass monotonic loading data (model 1). In Fig. 5b, the results of performing the same simulations are shown where the set of constants has been determined from the brass hysteresis data (model 2). In Fig. 5c, the hysteresis loops obtained for the two different material parameter sets are compared. It can be seen that utilizing the monotonic loading data for the determination of material constants instead of using the hysteresis data, does not produce any negative consequences. What is more, an excellent approximation is achieved in the initial part of the stress-stretch curve (Fig. 4b). In Fig. 5d, the result of one-dimensional, cyclic loading-unloading simulation is shown (model 1). 
(a)

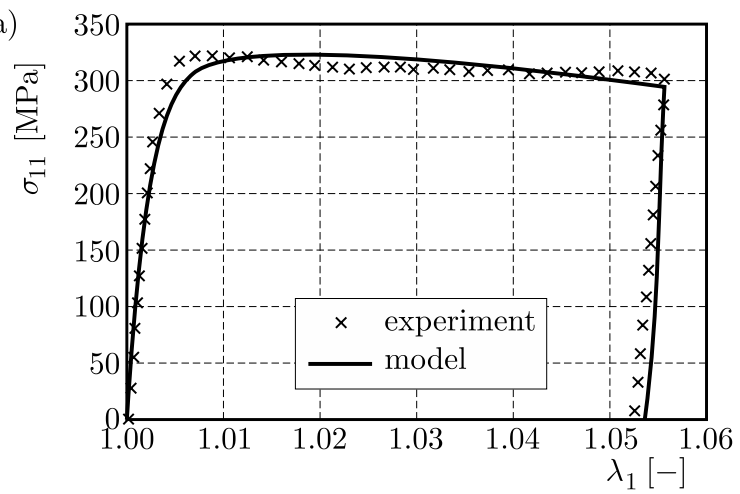

(c)

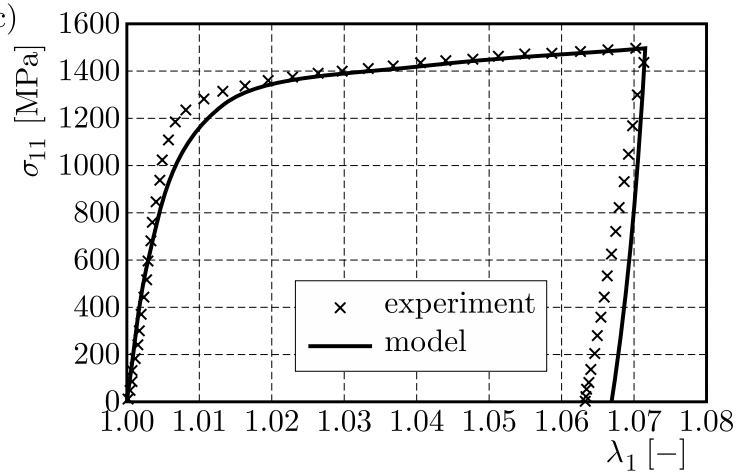

(e)

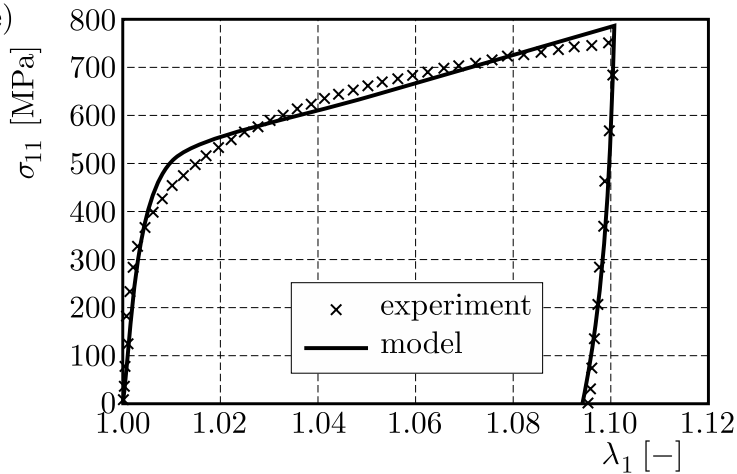

(b)

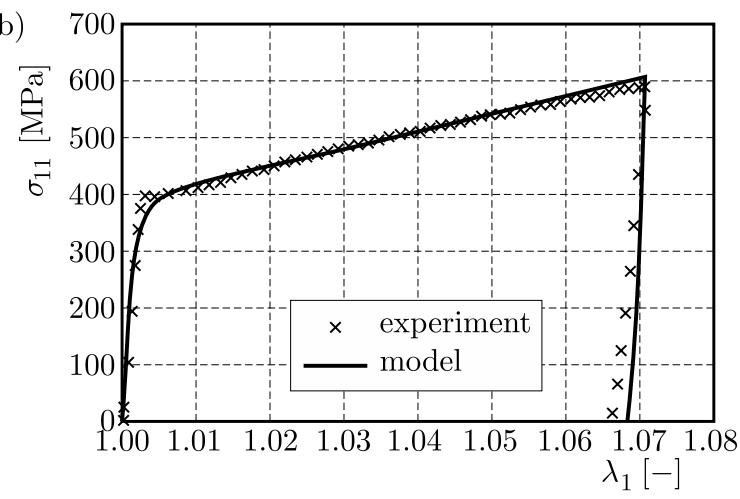

(d)

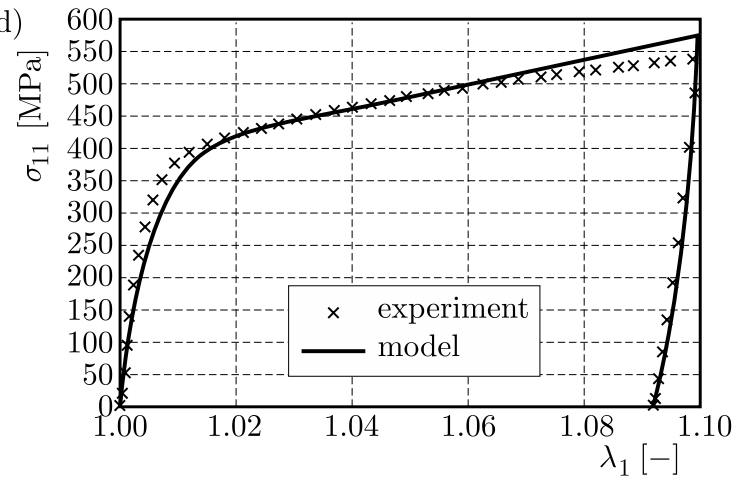

(f)

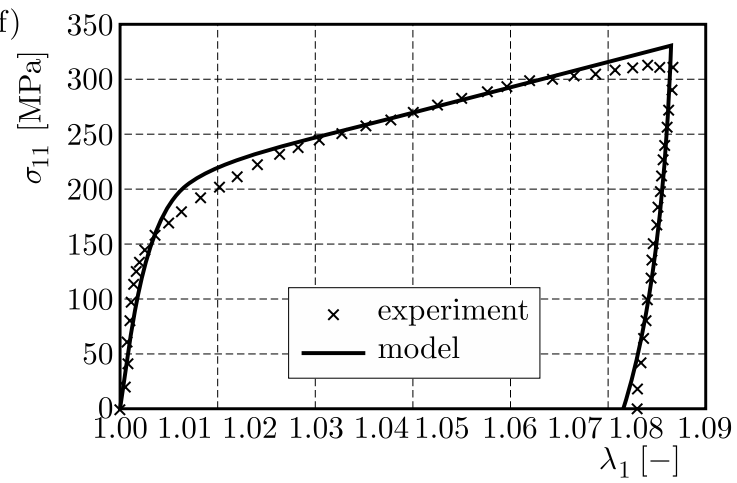

Fig. 3. Fitting of constitutive model predictions to experimental data: (a) copper, (b) Inconel No. 1, (c) Inconel No. 2, (d) brass, (e) NC steel, (f) aluminum
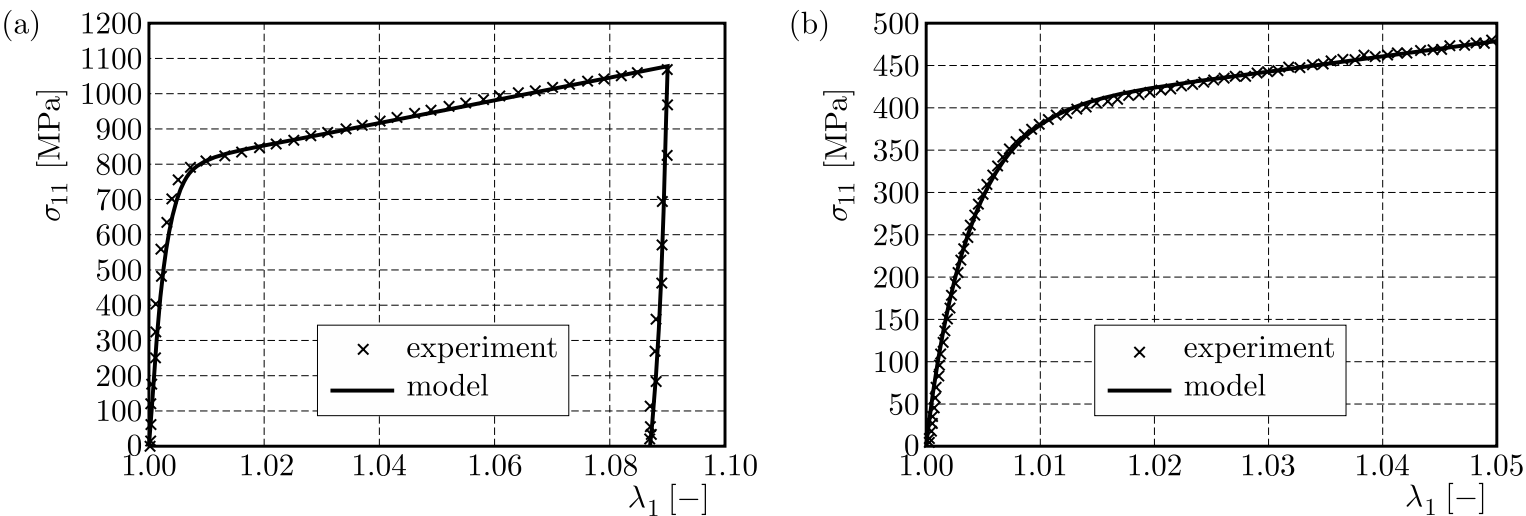

Fig. 4. Curve fitting results: (a) THA hysteresis data, (b) brass monotonic tension data 

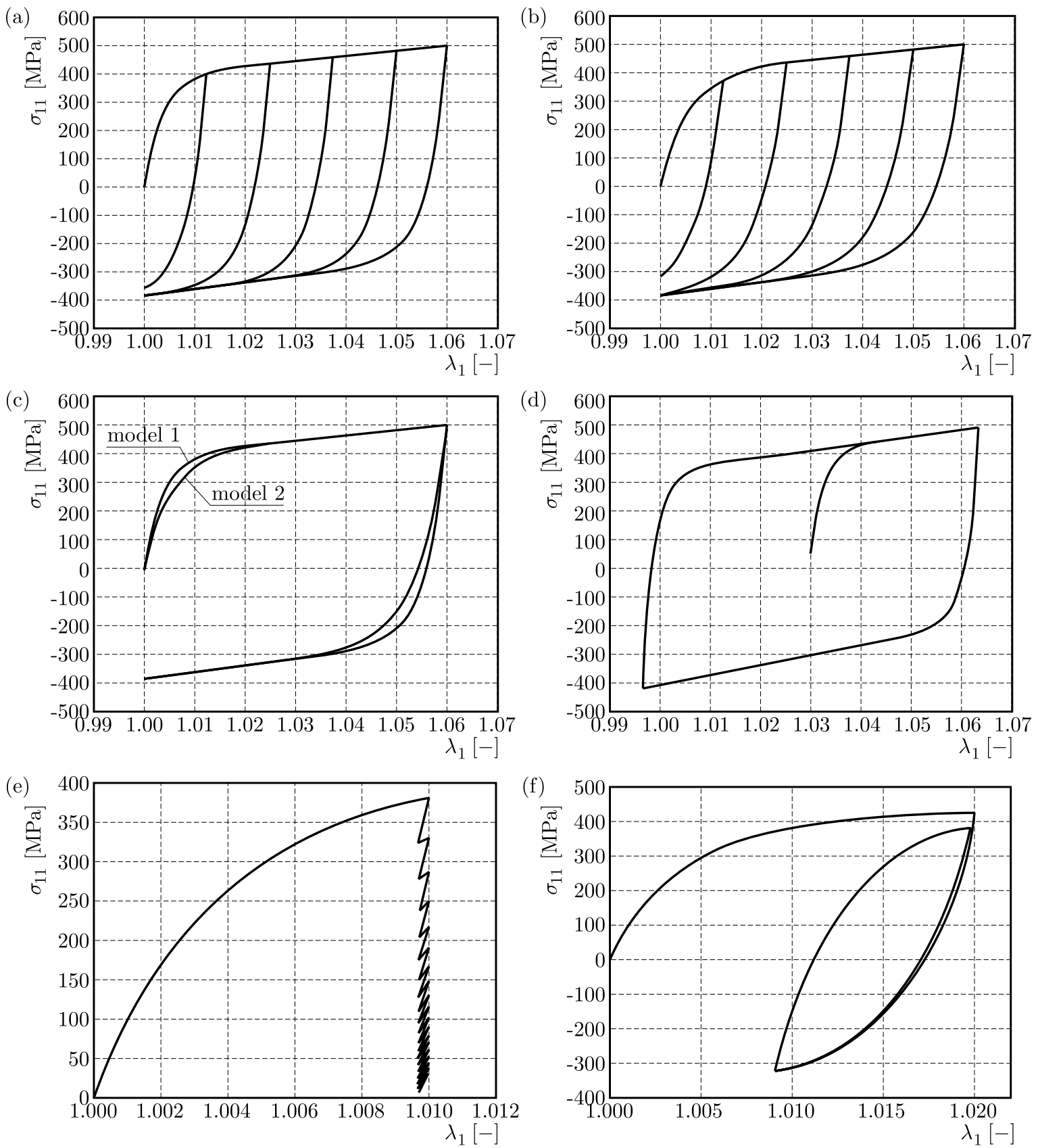

Fig. 5. Results for the brass deformation process simulation; (a) loading-unloading simulations for model 1, (b) loading-unloading simulations for model 2, (c) comparison of simulation results,

(d) hysteresis loop for brass, (e) cyclic unloading-loading with small stretch amplitude, (f) cyclic unloading-loading with large stretch amplitude

The response to cyclic loading-unloading stretch histories with a prestrain has been investigated (model 1). The stretch was monotonically increased to $\lambda_{1}^{\max }=1.01$. Subsequently, unloading was performed to $\lambda_{1}^{\min }=1.0097$ which was followed by loading until $\lambda_{1}^{\max }[-]$ was reached again. The unloading-loading cycle was repeated for 40 times. It can be seen in Fig. 5e that the unloading slope is larger than in the case of the following reloading, so that the hysteresis loop does not form and a stress decrease is observed. This defect is known for the group of endochronic models and was discussed by Rivlin (1981). The same simulation was repeated for a larger stretch amplitude, i.e. $\lambda_{1}^{\min }=1.009$ and $\lambda_{1}^{\max }=1.02$ which resulted in formation of a stress-stretch loop (Fig. 5f). It is concluded that for cyclic loadings with a prestrain, the model's applicability is limited to the case of large stress/stretch amplitudes. 
In Fig. 6 the results of two loading-unloading simulations, i.e. in tension and in compression, are plotted. The material constant values determined for brass (model 1) are utilized. It can be seen that the material responses in tension and compression are almost the same. What is more, the Bauschinger effect can be observed.

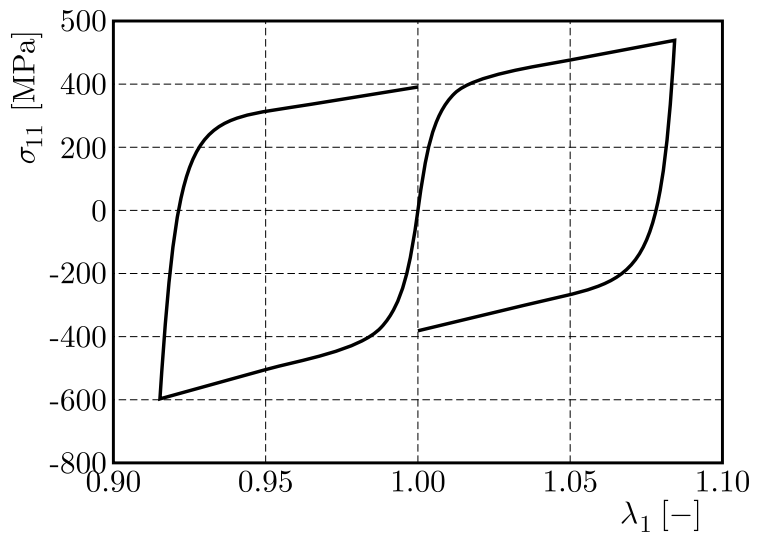

Fig. 6. Simulation of the Bauschinger effect in brass for tension and compression

(a)

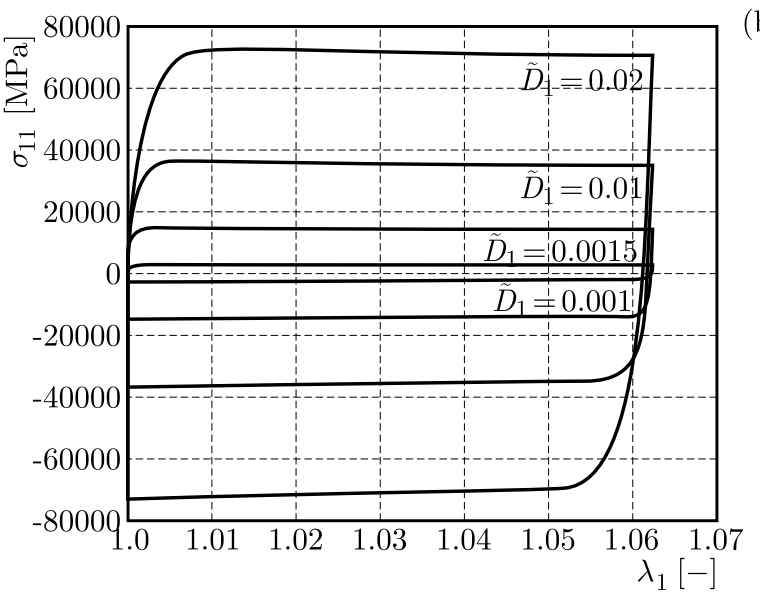

(b)

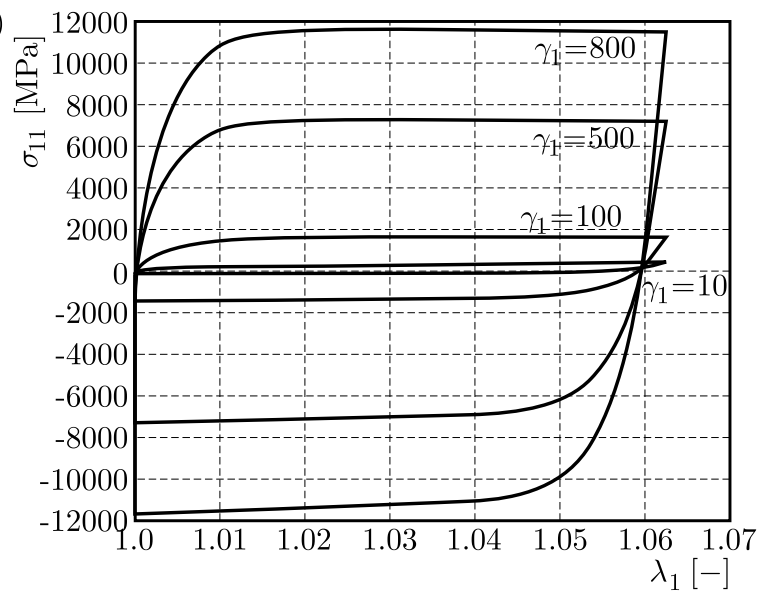

(c)

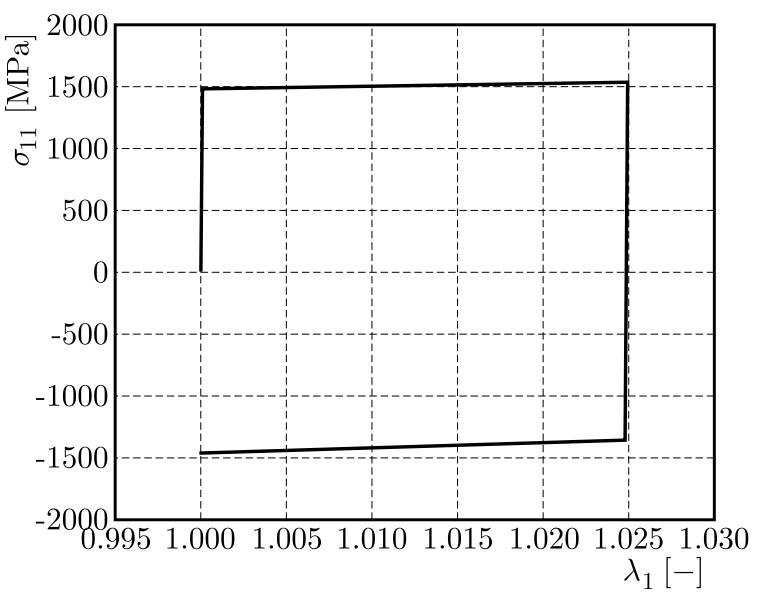

Fig. 7. Parametric study results; (a) hysteresis loops for different values of parameter $\widetilde{D}_{1}$, (b) hysteresis loops for different values of the coefficient $\gamma_{1}$, (c) the case of a rigid-perfectly plastic solid

The influence of the constant values has been investigated. In Fig. 7a, the uniaxial loading-unloading stress-stretch curves obtained for different values of the parameter $\widetilde{D}_{1}$ are shown. In Fig. 7b, the uniaxial loading-unloading curves generated for different values of the coefficient $\gamma_{1}$ 
are plotted. Some adjustments of the $\widetilde{D}_{1}$ value allow one to obtain the response of a rigid-perfectly plastic solid (Fig. 7c).

\section{Conclusions}

In this study, a finite strain formulation of plasticity without a yield surface has been presented and analyzed. The material parameter values are identified for a number of metallic materials. A good agreement between the theoretical predictions and the experimental data is achieved. It is found that the presented model can capture such behavior as indefinite elastic limit, strain hardening, strain softening and the Bauschinger effect. The model limitations are discussed as well. The algorithm for material constant evaluation has been presented and a parametric study conducted.

Acknowledgement

This work was supported by project INNOTECH-K2/IN2/27/182101/NCBR/13.

\section{References}

1. BaCKhaus G., 1976, Fließspannungen und Fließbedingung bei zyklischen Verformungen, ZAMM, $\mathbf{5 6}, 8,337-348$

2. Ilyushin A.A., 1963, Plasticity, Foundations of the General Mathematical Theory (in Russian), Academy of Sciences of the USSR, Moscow

3. Mróz Z., RodziK P., 1996, On multisurface and integral description of anisotropic hardening evolution in metals, European Journal of Mechanics - A/Solids, 15, 1, 1-28

4. Pipkin A.C., Rivlin R.S., 1965, Mechanics of rate-independent materials, ZAMP, 16, 313-327

5. Rivlin R.S., 1981, Some comments on the endochronic theory of plasticity, International Journal of Solids and Structures, 17, 231-248

6. Suchocki C., 2015, An internal-state-variable based viscoelastic-plastic model for polymers, Journal of Theoretical and Applied Mechanics, 53, 593-604

7. VAlanis K.C., 1971a, A theory of viscoplasticity without a yield surface, Part I: General theory, Archives of Mechanics, 23, 517-534

8. Valanis K.C., 1971b, A theory of viscoplasticity without a yield surface, Part II: Application to mechanical behavior of metals, Archives of Mechanics, 23, 535-551

9. VAlanis K.C., 1980, Fundamental consequences of a new intrinsic time measure, plasticity as a limit of the endochronic theory, Archives of Mechanics, 32, 171-191

10. ZALEWSKI R., 2009, Numerical method of Chaboche's model parameters identification basing on special granular structures experimental data, Modelling in Engineering, 38, 2, 309-319, (in Polish) 